Dermatology 2020;236:296-297

DOI: $10.1159 / 000504912$

\section{Dietary Intervention: An Essential Part of the Management of Patients Affected by Hidradenitis Suppurativa}

Claudio Marasca ${ }^{\mathrm{a}}$ Eleonora Cinelli ${ }^{\mathrm{a}}$ Maria Carmela Annunziata ${ }^{\mathrm{a}}$ Luigi Barrea $^{\text {b Silvia Savastano }}{ }^{\text {b }}$ Gabriella Fabbrocini ${ }^{\mathrm{a}}$

a Section of Dermatology, Department of Clinical Medicine and Surgery, University of Naples Federico II, Naples, Italy; ${ }^{\text {U Unit of }}$ Endocrinology, Dipartimento di Medicina Clinica e Chirurgia, Federico II University Medical School of Naples, Naples, Italy

Keywords

Acne inversa $\cdot$ Inflammation $\cdot$ Skin inflammation

Dear Editor,

We have read with great interest the article by Dempsey et al. [1] (2019) who carried out a cross-sectional survey on dietary alterations made by patients affected by hidradenitis suppurativa (HS). Their study highlights how avoidance of particular foods is a common management strategy attempted by HS patients [1].

In our opinion dietary patterns may have an important role in the management of HS, and the patients should be informed by experts about the most appropriate dietary plan for their clinical status. In line with our recent study, we suggest evaluation of individual nutritional status as an essential part in the management of HS patients [2]. In fact, in HS patients, the clinical severity (HS Sartorius score) was negatively and statistically significantly associated with both phase angle (PhA) values and adherence to the Mediterranean diet (MD) [2].

The $\mathrm{PhA}$ is a bioelectrical impedance analysis parameter identified as a prognostic marker of both morbidity and mortality in chronic inflammatory states [3]. The parameter is a sign of cellular integrity and of water distribution in body fluids $[4,5]$. PhA values are lower in a substantial group of inflammatory diseases, including also skin conditions such as psoriasis and HS [2, 6, 7].

The MD is a well-recognized nutritional tool that affects the systemic inflammatory balance and has long-lasting hormetic effects [8]. Previous studies have already underlined its role in the multidisciplinary management of other inflammatory cutaneous diseases. In 2018, Phan et al. [9] demonstrated how this dietary regimen can slow the progression of psoriasis, and it is advisable in pregnant women as it potentially decreases the risk of atopy in their offspring [10]. Compared to the control group, HS patients showed different dietary habits tending to a proinflammatory status characterized by a lower intake of complex carbohydrate, monounsaturated fatty acids, and n-3 polyunsaturated fatty acids, and a higher consumption of saturated fatty acid and n-6 polyunsaturated fatty acids [2]. Moreover, low adherers to the MD had a statistically significantly higher score in the HS Sartorius scale [2].

In addition, it has been previously shown that HS patients have a significantly higher prevalence of insulin resistance. We recently analysed homoeostasis model assessment of insulin resistance (HOMA-IR) in HS patients and healthy subjects with patients having higher values $(p=0.023)$. Moreover, we assessed the rate of glucose and insulin secretion after the oral glucose tolerance test. HS patients showed a significant difference regarding the insulin but not the glucose rate with respect to healthy subjects. HS subjects affected by insulin resistance showed a correlation with disease severity as well as with the glucose rate and BMI [11].

Therefore, dermatologists and nutritionists should have a multidisciplinary approach in HS patients in order to inform them about the appropriate diet plan, guiding their dietary habits and promoting a higher intake of foods with anti-inflammatory effects as in the MD.

In conclusion, in agreement with the study of Dempsey et al. [1], we underline once again the role of dietary patterns in the management of HS patients, focusing in particular on bioelectrical impedance analysis parameters (PhA) and MD diet adherence as a potential tool in the therapeutic intervention and prognostic evaluation of HS.

\section{Key Message}

Dietary patterns, Mediterranean diet adherence, and evaluation of bioelectrical impedance analysis parameters are central in hidradenitis suppurativa management.

\section{Disclosure Statement}

None of the contributing authors have any conflict of interest, including specific financial interests of relationships and affiliation, relevant to the subject matter or discussed materials in this paper.

\section{Funding Sources}

The authors have no funding sources to declare.

\section{References}

1 Dempsey A, Butt M, Kirby JS. Prevalence and impact of dietary avoidance among individuals with hidradenitis suppurativa. Dermatology. 2019 Nov:1-7. Epub ahead of print.

2 Barrea L, Fabbrocini G, Annunziata G, Muscogiuri G, Donnarumma M, Marasca C, et al. Role of nutrition and adherence to the Mediterranean diet in the multidisciplinary approach of hidradenitis suppurativa: evaluation of nutritional status and its association with severity of disease. Nutrients. 2018 Dec;11(1):E57. karger@karger.com

www.karger.com/drm

Karger
(C) 2019 S. Karger AG, Basel
Claudio Marasca, MD

Section of Dermatology and Venereology

Department of Clinical Medicine and Surgery, University of Naples Federico II

Via Pansini 5, IT-80131 Naples (Italy)

E-Mail claudio.marasca@gmail.com 
3 Norman K, Stobäus N, Zocher D, Bosy-Westphal A, Szramek A, Scheufele R, et al. Cutoff percentiles of bioelectrical phase angle predict functionality, quality of life, and mortality in patients with cancer. Am J Clin Nutr. 2010 Sep;92(3):612-9.

4 Siddiqui NI, Khan SA, Shoeb M, Bose S. Anthropometric predictors of bio-impedance analysis (BIA) phase angle in healthy adults. J Clin Diagn Res. 2016 Jun;10(6):CC01-04.

5 Barbosa-Silva MC, Barros AJ, Wang J, Heymsfield SB, Pierson RN Jr. Bioelectrical impedance analysis: population reference values for phase angle by age and sex. Am J Clin Nutr. 2005 Jul;82(1):49-52.

6 Barrea L, Macchia PE, Di Somma C, Napolitano M, Balato A, Falco A, et al. Bioelectrical phase angle and psoriasis: a novel association with psoriasis severity, quality of life and metabolic syndrome. J Transl Med. 2016 May;14(1):130.
7 Barrea L, Muscogiuri G, Laudisio D, Di Somma C, Salzano C, Pugliese $\mathrm{G}$, et al. Phase angle: a possible biomarker to quantify inflammation in subjects with obesity and 25(OH)D deficiency. Nutrients. 2019 Jul;11(8): 1747.

8 Martucci M, Ostan R, Biondi F, Bellavista E, Fabbri C, Bertarelli C, et al. Mediterranean diet and inflammaging within the hormesis paradigm. Nutr Rev. 2017 Jun;75(6):442-55.

9 Phan C, Touvier M, Kesse-Guyot E, Adjibade M, Hercberg S, Wolkenstein $\mathrm{P}$, et al. Association between Mediterranean anti-inflammatory dietary profile and severity of psoriasis: results from the NutriNet-Santé cohort. JAMA Dermatol. 2018 Sep;154(9):1017-24.

10 Amati F, Hassounah S, and Swaka A. The impact of Mediterranean dietary patterns during pregnancy on maternal and offspring health. Nutrients. 2019 May;11(5):1098.

11 Marasca C, Balato A, Annunziata MC, Cacciapuoti S, Fabbrocini G. Insulin resistance, mTOR and hidradenitis suppurativa. J Eur Acad Dermatol Venereol. 2019 Mar;33(3):e106-7. 